\title{
Doppler ultrasonographic evaluation of radial and ulnar artery diameters and blood flow, before and after percutaneous coronary interventions
}

\author{
Yasemin Gunduz ${ }^{1}$, Huseyin Gunduz ${ }^{2}$, Omer Faruk Ates ${ }^{1}$, Mahmut Ciner ${ }^{1}$, Ahmet Can \\ Cakmak $^{2}$, Cagla Akcay ${ }^{2}$, Ersin Ilguz ${ }^{2}$, Kahraman Cosansu ${ }^{2}$
}

${ }^{1}$ Radiology Department, ${ }^{2}$ Cardiology Department, Sakarya University Medical Faculty, Sakarya, Turkey

\begin{abstract}
Aim: Although the transforearm approach is considered a safe and effective option for percutaneous coronary intervention, the different characteristics of the radial and ulnar arteries deserve attention. This study aimed to evaluate radial (RA) and ulnar artery (UA) diameter and blood flow parameters changes after catheterization. Material and method: A total of 328 patients were enrolled. Their artery (171 RA and 157 UA) diameter and flow parameters [peak systolic velocity (PSV), end-diastolic volume (EDV) and pulsatility index (PI)] were evaluated before and after catheterisation. Results: After RA catheterization, the diameters and PSV decreased in the RA (from $2.71 \pm 0.66$ to $2.47 \pm 0.51, \mathrm{p}=0.007$; from $44.7 \pm 8.3$ to $33.9 \pm 9.5, \mathrm{p}=0.021$ ) and increased in the UA (from $2.49 \pm 0.83$ to $2.59 \pm 0.58, \mathrm{p}=0.033$; from $48.3 \pm 11.9$ to $59.6 \pm 11.0, \mathrm{p}<0.001$ ). After UA catheterization, the diameters and PSV decreased in UA (from $2.53 \pm 0.65$ to $2.49 \pm 0.77 \mathrm{~mm}, \mathrm{p}=0.173$; from $51.2 \pm 13.1$ to $44.3 \pm 10.7 \mathrm{~cm} / \mathrm{s}$, $\mathrm{p}=0.081$ ) and increased in RA (from $2.67 \pm 0.54$ to $2.76 \pm 0.43 \mathrm{~mm}, \mathrm{p}=0.040$; from $41.8 \pm 10.3$ to $48.6 \pm 7.9 \mathrm{~cm} / \mathrm{s}, \mathrm{p}=0.054$ ). Conclusions: The marked increase in the diameter and PSV of the non-catheterized artery may indicate compensatory changes in the hand arterial network. Acute wall changes may have led to an increase in total wall thickness and a modest decrease in lumen size and blood flow velocity in the catheterized artery.

Keywords: Doppler ultrasonography; radial artery; ulnar artery; coronary angiography; percutaneous coronary intervention
\end{abstract}

\section{Introduction}

The radial artery (RA) and ulnar artery (UA) are commonly the preferred access sites for coronary angiography (CAG) and percutaneous intervention (PCI). While the transforearm approach is considered a safe and ef-

Received 12.12.2020 Accepted 05.06.2021

Med Ultrason

2022, Vol. 24, No 1, 52-57

Corresponding author: Yasemin Gunduz, MD, Associate Professor Sakarya University Medical Faculty, Radiology Department, Istiklal mah, 342 sokak, Hakkı Bey konakları, 54100, Serdivan, Sakarya, Turkey Phone: 0905322769390

E-mail: dryasemingunduz@yahoo.com fective option for PCI, the different anatomical and flow characteristics of the RA and UA deserve attention [1,2]. Both arteries form dense anastomotic arches that provide sufficient blood flow to the hand. This relationship is less clear at the level of the wrist because the UA gives off multiple branches in the forearm, whereas the RA serves mainly as an arterial conduit to the hand [3]. In addition, the closed-loop nature of the radio-ulnar circulation also provides an opportunity for the potential alteration of flow characteristics in one artery by altering flow in the other artery [4].

The arterial velocity increases in response to compression or occlusion of the opposite artery at the wrist in normal subjects. Thus, when the RA is compressed at the level of the wrist, UA blood flow increases and an increase in UA velocity after RA compression is a sign of adequate collateral perfusion. Similarly, when the UA 
is compressed, RA blood flow increases, which indicates a functional continuity between radial and ulnar circulation in the hand [5].

The first invasive stage of PCI procedures is the catheterization of the RA or UA. In many studies, despite conflicting results [6], changes in blood flow parameters and diameters of the RA and UA after reciprocal compression/occlusion have been investigated in the early or late periods of the procedure using Doppler ultrasonography (DUS) [7-10]. However, blood flow characteristics and diameter changes in both arteries (catheterized and non-catheterized) before and after RA or UA catheterization for CAG and/or PCIs have not been examined or compared during the early period.

Our study hypothesis focusing on RA and UA catheterization (including cannulation, decannulation, sheath insertion, sheath removal and 4 hours of gradually decreasing compression) was that this procedure will cause changes in the structure and hemodynamics of both arteries. For this reason, the aim of our study was to evaluate the structural and hemodynamic changes in RA and UA after catheterization by using DUS.

\section{Materials and methods}

\section{Study population}

Between January 2018 and August 2020, a total of 328 patients who underwent CAG and/or PCI were included in the study: 171 patients via a transradial approach (TRA), 157 patients via a transulnar approach (TUA). All procedures were performed through the right UA and RA. DUS was carried out before procedure (maximum 48 hours before catheterisation) and 5-48 hours after compression bandage was removed.

The main indication for TUA or TRA was a wider and easily palpable pulse. The demographic and clinical characteristics (age, gender, systolic and diastolic blood pressure, clinical diagnoses, fasting blood glucose, lipid profile, history of diabetes mellitus, hypertension, smoking, chronic kidney disease, and drug use) were recorded.

Study exclusion criteria were as follows: $<18$ or $>80$ years of age, previous CAG and/or PCI by TRA or TUA, severe heart failure, hemodynamic impairment, uncontrolled hypertension, acute or chronic renal failure or hepatic diseases, chronic pulmonary disease, bleeding diathesis, severe skeletal forearm deformities, peripheral artery disease (i.e. Raynaud's disease), or previous coronary artery bypass surgery using an RA graft.

Sakarya University Medical Faculty Ethics Committee approved the study protocol and each patient signed a written informed consent form.

\section{Catheterization technique}

The right forearm was abducted and placed on the catheterization table with hyperextension of the wrist. After a local anesthetic injection, arterial puncture was achieved. Following palpation of the site of maximal pulse prominence, using the Seldinger technique, a 0.025inch straight guidewire was passed through a needle. The needle was removed and a $5 \mathrm{~F}$ or $6 \mathrm{~F}$ hydrophilic sheath was placed over the guidewire. A spasmolytic cocktail (100-200 $\mu \mathrm{g}$ of Perlinganit and $12.5 \mu \mathrm{g}$ of diltiazem, if required) and heparin (5000 IU bolus in case of diagnostic catheterization or 70-100 IU/kg in case of PCI) were infused intra-arterially through the sheath. For diagnostic purposes, conventional 5F Tiger catheters (Terumo Corp., Tokyo, Japan), and for PCI 5F or 6F (rarely, in complex PCI) extra back-up, Judkins, or Amplatz guiding catheters were used. After CAG or PCI, the arterial sheath was removed immediately following cardiac catheterization completion. Local haemostasis was achieved using a pressure bandage (Terumo, Tokyo, Japan) inflated at the puncture site with 15 to $20 \mathrm{~mL}$ of air. The pressure used for UA compression was higher than that for the RA, as there is no bone structure to compress the UA at the bottom and the UA is a deeper vessel. These bandages were removed after 4 hours and the patients were discharged within 48 hours of procedure completion. Catheterization procedures for both arteries were performed by the same operator (H.G.) with more than 10 years of experience and over 7000 catheterizations.

\section{Ultrasonographic evaluation}

All DUS examinations were performed by the same radiologist (Y.G) with more than 20 years of experience, in DUS using an Aplio 400 Doppler USG system (Toshiba, Tokyo, Japan) with a 7.2-14 MHz multi-frequency linear array transducer.

The lumen diameters, peak systolic velocity (PSV), end-diastolic velocity (EDV), pulsatility index (PI) and resistive index (RI) were measured at the wrist level. The sagittal diameter of the RA and UA were measured at the diastolic phase (from intima to intima) in a short-axis section. The ultrasound examination was performed at room temperature $\left(22\right.$ and $\left.23^{\circ} \mathrm{C}\right)$, the examination room temperature being recorded before each DUS.

\section{Statistical analysis}

An independent Student's t-test was used to compare data for continuous variables which were expressed as mean and standard deviation [age, blood pressure, blood biochemistry measurements (fasting glucose, LDL cholesterol, and triglycerides)]. The diameter and Doppler flow of the RA and UA before and after the procedure were compared using a paired t-test. Categorical variables expressed as numbers and percentages (gender, clin- 
ical diagnosis, diabetes mellitus, hypertension, smoking, dyslipidaemia, and chronic kidney disease) were compared by Pearson's chi-squared test or Fisher's exact test, when appropriate.

All analyses were performed using the SPSS Statistics software package for Windows, version 22.0 (SPSS Inc., Chicago, IL, USA). A value of $\mathrm{P}<0.05$ was considered statistically significant.

Considering the RA and UA catheterizations as two different access methods (from 2020 Medcalc statistical software, Ostend, Belgium), the measurements were plotted using the Bland-Altman method to determine whether the differences showed a systematic distribution around zero and its prevalence.

\section{Results}

A total of 328 consecutive adult patients undergoing selective transradial (171) or transulnar (157) catheteri- zation procedures for $\mathrm{CAG}$ and $\mathrm{PCI}$ were included in this study. The demographic, clinical, laboratory and procedural time data of these groups are detailed in Table I.

Ultrasonographic measurements of radial and ulnar arteries before and after catheterization are specified in table II.

Comparing the right and left RA and UA preprocedural ultrasound parameters, no significant differences were found concerning the diameters (left RA and $2.64 \pm 0.74$ $\mathrm{mm}$, left UA $2.47 \pm 0.98 \mathrm{~mm}$ ) or Doppler flow measurements (all $\mathrm{p}>0.05$ ). The RA was the dominant forearm artery in most cases (198 patients, 62.2\%).

Due to the detection of significant changes in the diameter and PSV of both arteries after catheterization, the compliance levels examined using the Bland-Altman method showed no difference. The plots showed a systematic distribution of the differences around zero from the distribution graphs and a clear relationship between the differences and the means (fig 1).

Table I. Demographic, clinical and laboratory findings of study patients

\begin{tabular}{|c|c|c|c|c|}
\hline & $\begin{array}{l}\text { All patients } \\
(\mathbf{n}=328)\end{array}$ & $\begin{array}{l}\text { RA catheterization } \\
(n=171)\end{array}$ & $\begin{array}{l}\text { UA catheterization } \\
(n=157)\end{array}$ & p values \\
\hline Age (years) & $61 \pm 17$ & $63 \pm 11$ & $59 \pm 20$ & 0.077 \\
\hline Gender $(F)$ & $174(53)$ & $89(52)$ & $85(54)$ & 0.701 \\
\hline \multicolumn{5}{|l|}{ Diagnosis } \\
\hline Acute coronary syndrome & $121(37)$ & $60(35)$ & $61(39)$ & 0.312 \\
\hline Stable angina pectoris & $177(54)$ & $94(54.9)$ & $83(52.8)$ & 0.879 \\
\hline Miscellaneous & $30(9.1)$ & $14(8.1)$ & $16(10.2)$ & 0.183 \\
\hline Hypertension & $131(40)$ & $70(40.9)$ & $61(38.8)$ & 0.619 \\
\hline Right arm preprocedure systolic/diastolic BP (mmHg) & $134 / 84$ & $132 / 83$ & $136 / 86$ & 0.416 \\
\hline Right arm postprocedure systolic/diastolic BP (mmHg) & $135 / 82$ & $133 / 81$ & $138 / 83$ & 0.097 \\
\hline Diabetes mellitus & $59(17.9)$ & $33(19.2)$ & $26(16.5)$ & 0.115 \\
\hline Dyslipidemia & $128(39)$ & $71(41.5)$ & $57(36.3)$ & 0,092 \\
\hline Smoking & $121(36)$ & $68(39.7)$ & $53(33.7)$ & 0.053 \\
\hline Chronic kidney disease & $30(9.1)$ & $13(7.6)$ & $17(10.8)$ & 0.182 \\
\hline \multicolumn{5}{|l|}{ Procedural time parameters (min) } \\
\hline Arterial access time & $4.6 \pm 2.3$ & $4.4 \pm 3.1$ & $4.7 \pm 2.9$ & $>0.05$ \\
\hline Coronary angiography time & $9.3 \pm 5.8$ & $8.8 \pm 4.3$ & $9.7 \pm 6.1$ & $>0.05$ \\
\hline Elective PCI time & $21.8 \pm .9 .1$ & $20.9 \pm 11.3$ & $22.7 \pm 10.1$ & $>0.05$ \\
\hline Total procedural time & $31.6 \pm .14 .7$ & $31.2 \pm 10.9$ & $32.1 \pm 13.1$ & $>0.05$ \\
\hline \multicolumn{5}{|l|}{ Medications (n) } \\
\hline ACE inhibitors/ARBs & 135 & 75 & 60 & 0.553 \\
\hline Amlodipin & 62 & 33 & 29 & 0.817 \\
\hline Metoprolol & 85 & 47 & 38 & 0.376 \\
\hline Diuretics & 52 & 30 & 22 & 0.408 \\
\hline Digoxin & 19 & 12 & 7 & 0.279 \\
\hline Statin & 109 & 56 & 53 & 0.911 \\
\hline
\end{tabular}

The results are expressed as mean \pm standard deviation or number (\%). BP: blood pressure; ACE: angiotensin-converting enzyme; ARBs: angiotensin II receptor blockers; LDL: low-density lipoprotein; Diuretics; PCI: percutaneous coronary intervention. 
Table II. Ultrasonographic measurements of radial and ulnar arteries before and after catheterization

\begin{tabular}{lllllll}
\hline & \multicolumn{2}{l}{ RA catheterization } & \multicolumn{3}{l}{ UA catheterization } \\
\cline { 2 - 7 } & Pre & Post & p values & Pre & Post & p values \\
\hline RA diameter $(\mathrm{mm})$ & $2.71 \pm 0.66$ & $2.47 \pm 0.51$ & 0.007 & $2.67 \pm 0.54$ & $2.76 \pm 0.43$ & 0.040 \\
UA diameter $(\mathrm{mm})$ & $2.49 \pm 0.83$ & $2.59 \pm 0.58$ & 0.033 & $2.53 \pm 0.65$ & $2.49 \pm 0.77$ & 0.173 \\
RA PSV & $44.7 \pm 8.3$ & $33.9 \pm 9.5$ & 0.021 & $41.8 \pm 10.3$ & $48.6 \pm 7.9$ & 0.054 \\
UA PSV & $48.3 \pm 11.9$ & $59.6 \pm 11.0$ & $<0.001$ & $51.2 \pm 13.1$ & $44.3 \pm 10.7$ & 0.081 \\
UA EDV & $9.1 \pm 3.0$ & $9.4 \pm 2.0$ & 0.481 & $8.8 \pm 1.6$ & $8.5 \pm 2.2$ & 0.169 \\
RA PI & $4.1 \pm 1.9$ & $3.9 \pm 1.4$ & 0.713 & $4.4 \pm 0.7$ & $4.3 \pm 1.2$ & 0.791 \\
UA PI & $4.4 \pm 2.7$ & $4.0 \pm 2.3$ & 0.059 & $4.3 \pm 1.4$ & $4.7 \pm 1.9$ & 0.674 \\
RA RI & $0.78 \pm 0.09$ & $0.75 \pm 0.06$ & 0.177 & $0.77 \pm 0.23$ & $0.80 \pm 0.19$ & 0.328 \\
UA RI & $0.80 \pm 0.11$ & $0.83 \pm 0.17$ & 0.269 & $0.82 \pm 0.12$ & $0.79 \pm 0.24$ & 0.552 \\
\hline
\end{tabular}

The results are expressed as mean \pm standard deviation. UA: ulnar artery; RA: radial artery; PSV: peak systolic velocity; EDV: end-diastolic velocity; PI: pulsatility index; RI: Resistive index

\section{Discussion}

In our study, we demonstrated the appearance of changes in the arterial diameter and blood flow velocity in both RA and UA after catheterization. In addition, while the diameter and PSV decreased in the catheterized artery, the diameter and PSV of the non-catheterized artery of the same arm increased.

Although the catheterization process is the same for both arteries, the effect of compression on the catheterized and ipsilateral non-catheterized artery may be different. Compression of the deeply located UA is more difficult; therefore, the pressure of the bandage is kept higher for better compression of the UA. The flow velocity of the adjacent artery increased less (compared to RA catheterization) due to the less reduction in UA diameter and flow velocity. In addition, it may be thought that the reduction in the diameter and flow velocity of the UA, which has fewer alpha receptors and less sympathetic activity and therefore less spasm tendency, may contribute to this result. The effect of haemostasis attempts after catheterization may also be less pronounced in the UA for these reasons. In the RA, which is more easily compressed due to its superficial course, it has more alpha receptors and a higher tendency to spasm, the diameter and velocity decreased significantly after catheterization. Thus the velocity and diameter of the adjacent UA increased significantly, similar to previous study reports $[3,11,12]$.

It was known that RA flow is significantly lower than UA flow and peripheral resistance at the radial portion of the vascular bed is significantly higher comparing to the ulnar portion $[5,13,14]$. Doscher et al reported that the mean diameters of the RA and UA did not differ significantly; however, PSV of the RA was lower than that of the UA [15].
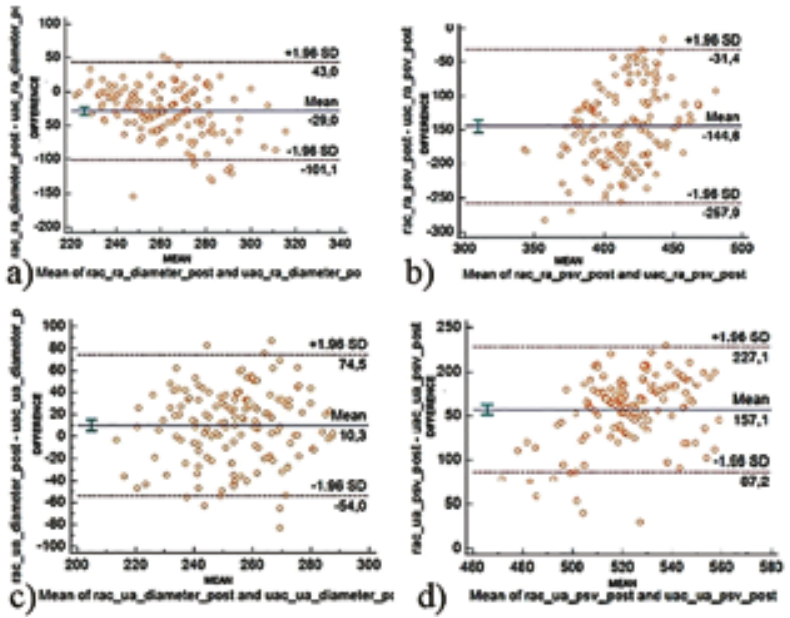

Fig 1. The Bland-Altman plots showing the compliance levels after RA and UA catheterization. The mean and standard deviation of the differences between the diameter (A) and PSV (B) changes in the RA and the diameter (C) and PSV (D) changes in the UA are given as $\pm 1.96 \mathrm{SD}$ and the distribution is seen to be around the mean.

Indeed, as determined in our study, the increase in UA flow velocity after RA catheterization was more pronounced than that of the RA after UA catheterization. The higher basal UA flow velocity compared to that of the RA may contribute to the more pronounced increase in flow velocity of the UA. In addition, although a diameter decrease in the UA after UA catheterization was determined, it was not as apparent as the difference after RA catheterization in the RA. This result may be another reason why the increase in RA PSV after UA catheterization was not as marked and significant as the increase in UA PSV after RA catheterization. Our results were in line with those of previous studies [2,16], indicating an increase in the RA diameter and blood flow after transient UA compression. In our study, the fact that the 
RA diameter was greater than that of the UA may have affected these results. The fact that the basal UA flow velocity was higher than the basal RA flow velocity (as in the left arm) should not be ignored since the results obtained in our study may be related to the diameter and flow characteristics found during basal measurements (in other words, already existing).

Considering the data obtained, our study showed a significant increase in blood flow rates in the UA in cases with RA catheterization, indicating a functional continuity between the radial and ulnar circulation in both hands. Changes in the inner diameter of an artery can affect arterial blood flow rates by causing changes in arterial wall properties. This equation shows a strong and inverse relationship between the blood flow velocity of one artery and the internal diameter of the other artery in the same arm. Therefore, the decrease in vessel lumen diameter of the catheterized artery may be associated with the increase in the flow velocity of the other artery in the same arm. With RA catheterization, the distal perfusion pressure of the capillary bed decreases, causing an increase in the pressure gradient between the UA and the capillary bed, resulting in increase of UA blood flow [17,18].

Arterial cannulation/compression or catheterization results in a decrease in blood flow and sometimes occlusion of the catheterized artery in the early or late periods. If the radial/ulnar artery is narrowed or occluded, distal perfusion of the capillary bed will be decreased and blood flow in the ipsilateral artery will be increased. Increases in the diameter and flow rates of the other arteries in patients developing stenosis or RA occlusion and stenosis or UA occlusion ensure sufficient blood supply to the hand and protect the hand against ischemic events. Significant increases in the diameter, velocity, and volume flow of the arteries during reciprocal compression/ cannulation or catheterization of the two arteries in different studies, including our study, support this assumption $[19,20]$.

It has been demonstrated that RA cannulation would decrease RA blood flow immediately after catheter placement and the compensatory increase in UA blood flow might quickly commence. Significant changes continued after 24 hours from the beginning of monitoring [18,21]. Zhenxian et al investigated the impact of transradial coronary procedures on the RA by ultrasonography. The PSV and diameter of the right RA were measured before, the first day and the first month after transradial coronary interventions. They found that the diameter of the RA decreased on the first day after the procedure and this decrease continued in the control performed 1 month later [22]. Abe et al found that a slight decrease in RA diameter continued even 3 months after the transradial intervention; however, they reported that this decrease did not reach statistical significance [8]. Madssen et al found a significant reduction in the right RA diameter compared to left RA diameter at a control USG examination 12 months after CAG via the right RA [9].

Our study has some limitations. This was a singlecentre study with a nonrandomized design. Larger, randomized, multi-centre and prospective studies are needed to identify changes in diameter and blood flow parameters using DUS in patients undergoing coronary angiography/ PCI to confirm our results. Since all DUS examinations were realized by the same radiologist, and the examinations were carried out in a short period before and after the procedure, the intraobserver variation analysis was not performed in this study. In addition, in our study, only the early effects of catheterization in patients who underwent PCI (patients are generally discharged within 48 hours after the procedure and it is thought that the effects of catheterization in the long period may be reversible) were evaluated.

\section{Conclusion}

The data obtained from our study suggest that catheterization (starting with cannulation, ending with the removal of the compression bandage after 4 hours) causes vascular wall changes in the catheterized artery. In our opinion, while the procedure causes a decrease in diameter and blood flow velocity through vascular wall changes and arterial compression in the catheterized artery, it protects the arm against ischemia by causing an increase in the compensatory diameter and blood flow in the other artery of the same arm. In addition, the UA can be safely used as an alternative to the RA catheterization.

\section{Conflict of interest: none}

\section{References}

1. Kar S. Transulnar cardiac catheterization and percutaneous coronary intervention: techniques, transradial comparisons, anatomical considerations, and comprehensive literature review. Catheter Cardiovasc Interv 2017;90:1126-1134.

2. Kaplanoglu H, Dinc E. Diameters and Flow Characteristics of Forearm Arteries in the Preoperative Period Using Doppler Ultrasonography in Healthy Individuals. Iran J Radiol 2018;15:e12904.

3. Marchese RM, Geiger Z. Anatomy, Shoulder and Upper Limb, Forearm Radial Artery. StatPearls Publishing, 2021.

4. Pancholy SB, Heck LA, Patel T. Forearm arterial anatomy and flow characteristics: a prospective observational study. J Invasive Cardiol 2015;27:218-221.

5. Kim SY, Lee JS, Kim WO, Sun JM, Kwon MK, Kil HK. Evaluation of radial and ulnar blood flow after radial artery 
cannulation with 20- and 22-gauge cannulae using duplex Doppler ultrasound. Anaesthesia 2012;67:1138-1145.

6. Tonks AM, Lawrence J, Lovie MJ. Comparison of ulnar and radial arterial blood-flow at the wrist. J Hand Surg Br 1995;20:240-242.

7. Ruengsakulrach P, Brooks M, Hare DL, Gordon I, Buxton BF. Preoperative assessment of hand circulation by means of Doppler ultrasonography and the modified Allen test. J Thorac Cardiovasc Surg 2001;121:526-531.

8. Abe S, Meguro T, Naganuma T, Kikuchi Y. Change in the diameter of the radial artery transradial intervention using a 6 French system in Japanese patients. J Invasive Cardiol 2001;13:573-575.

9. Madssen E, Haere P, Wiseth R. Radial artery diameter and vasodilatory properties after transradial coronary angiography. Ann Thorac Surg 2006;82:1698-1702.

10. Peruga JP, Peruga JZ, Kasprzak JD, et al. Ultrasound evaluation of forearm arteries in patients undergoing percutaneous coronary intervention via radial artery access: results of one-year follow-up. Kardiol Pol 2015;73:502-510.

11. Mason PJ, Shah B, Tamis-Holland JE, et al; American Heart Association Interventional Cardiovascular Care Committee of the Council on Clinical Cardiology; Council on Cardiovascular and Stroke Nursing; Council on Peripheral Vascular Disease; Council on Genomic and Precision Medicine. An Update on Radial Artery Access and Best Practices for Transradial Coronary Angiography and Intervention in Acute Coronary Syndrome: A Scientific Statement From the American Heart Association. Circ Cardiovasc Interv 2018;11:e000035.

12. He GW, Yang CQ. Characteristics of adrenoceptors in the human radial artery: clinical implications. J Thorac Cardiovasc Surg 1998;115:1136-1141.

13. Brzezinski M, Luisetti T, London MJ. Radial artery cannulation:a comprehensive review of recent anatomic and physio-logic investigations. Anesth Analg 2009;109:17631781.

14. Zabad MN, Janzer S, George JC. Radial and Ulnar Artery Occlusion Following Transradial and Transulnar Coronary Intervention Requiring Endovascular Revascularization. Cath Lab Digest 2019;27:6.
15. Doscher W, Viswanathan B, Stein T, Margolis IB. Hemodynamic assessment of the circulation in 200 normal hands. Ann Surg 1983;198:776-779.

16. Yilmaztepe MA, Yilmaz E. Effect of transient ulnar artery compression on radial artery diameter. Exp Ther Med 2018;16:3735-3739.

17. Beniwal S, Bhargava K, Kausik SK. Size of distal radial and distal ulnar arteries in adults of southern Rajasthan and their implications for percutaneous coronary interventions. Indian Heart J 2014;66:506-509.

18. Kim EJ, Soh S, Kim SY, et al. Impact of Diabetes Mellitus on Radial and Ulnar Arterial Vasoreactivity after Radial Artery Cannulation: A Randomized Controlled trial. Int J Med Sci 2016;13:701-707.

19. Saito S, Ikei H, Hosokawa G, Tanaka S. Influence of the ratio between radial artery inner diameter and sheath outer diameter on radial artery flow after transradial coronary intervention. Catheter Cardiovasc Interv 1999;46:173-178.

20. Hahalis G, Aznaouridis K, Tsigkas G, et al. Radial Artery and Ulnar Artery Occlusions Following Coronary Procedures and the Impact of Anticoagulation: ARTEMIS (Radial and Ulnar ARTEry Occlusion Meta-Analys IS) Systematic Review and Meta-Analysis. J Am Heart Assoc 2017;6:e005430.

21. Brodman RF, Hirsh LE, Frame R. Effect of radial artery harvest on collateral forearm blood flow and digital perfusion. J Thorac Cardiovasc Surg 2002;123:512-516.

22. Yan Z, Zhou Y, Zhao Y, Zhou Z, Yang S, Wang Z. Impact of transradial coronary procedures on radial artery. Angiology 2010;61:8-13.

23. Huzjan R, Brkljacić B, Delić-Brkljacić D, Biocina B, Sutlić Z. B-mode and color Doppler ultrasound of the forearm arteries in the preoperative screening prior to coronary artery bypass grafting. Coll Antropol 2004;28 Suppl 2:235-241.

24. Ashraf T, Panhwar Z, Habib S, Memon MA, Shamsi F, Arif J. Size of radial and ulnar artery in local population. J Pak Med Assoc 2010;60:817-819.

25. Yan ZX, Zhou YJ, Zhao YX, Zhou ZM, Yang SW, Wang ZJ. Anatomical study of forearm arteries with ultrasound for percutaneous coronary procedures. Circ J 2010;74:686692. 